\title{
A Hybrid Algorithm of Evolution and Simplex Methods Applied to Global Optimization
}

Hsin-Chuan Kuo

Associate Professor, Institute of Systems Engineering and Naval Architecture, National Taiwan Ocean University, 2. Pei-Ning Road, Keelung, Taiwan 202, R.O.C, khc@ntou.edu.tw

Jiang-Ren Chang

Professor, Institute of Systems Engineering and Naval Architecture, National, Taiwan Ocean University, 2. Pei-Ning Road, Keelung, Taiwan 202, R.O.C.

Kai-Shun Shyu

Doctorate Candidate, Institute of Systems Engineering and Naval Architecture, National Taiwan Ocean University, 2. Pei-Ning Road, Keelung, Taiwan 202, R.O.C.

Follow this and additional works at: https://jmstt.ntou.edu.tw/journal

Part of the Engineering Commons

\section{Recommended Citation}

Kuo, Hsin-Chuan; Chang, Jiang-Ren; and Shyu, Kai-Shun (2004) "A Hybrid Algorithm of Evolution and Simplex Methods Applied to Global Optimization," Journal of Marine Science and Technology. Vol. 12: Iss. 4, Article 7.

DOI: $10.51400 / 2709-6998.2248$

Available at: https://jmstt.ntou.edu.tw/journal/vol12/iss4/7

This Research Article is brought to you for free and open access by Journal of Marine Science and Technology. It has been accepted for inclusion in Journal of Marine Science and Technology by an authorized editor of Journal of Marine Science and Technology. 


\title{
A HYBRID ALGORITHM OF EVOLUTION AND SIMPLEX METHODS APPLIED TO GLOBAL OPTIMIZATION
}

\author{
Hsin-Chuan Kuo*, Jiang-Ren Chang**, and Kai-Shun Shyu***
}

Key words: evolution algorithm, simplex method, global optimization.

\begin{abstract}
In this paper, a hybrid method combining the evolution and simplex algorithms is proposed to deal with the global optimization problems of two-dimensional multi-minimum functions. Basically, the simplex method offers a search scheme without the gradient information and thus, it owns the merit of a better search speed for a local optimization problem and results in the deficiency of searching ability for the global one. In contrast, the evolution method has the better searching ability for the global problem but needs much more time. Therefore, the proposed hybrid method adopts the search technique of the simplex method and the concept of all population information. For an $n$-dimensional problem, the populations of equal to or greater than $(n+1)$ are taken with their all information of the respective generation to decide the next searching point. The proposed method has a better searching ability for the global optimization problem because this hybrid method has the characteristics of intensity and diversity during the evolution of populations moving stage. The searching ability for the global optimum is demonstrated by a benchmark testing example of multi-minimum function. Finally, several testing examples show that the success rate of global minimization approaches to $98 \%$.
\end{abstract}

\section{INTRODUCTION}

An effective optimization algorithm is dependent on its searching ability for global optimum solution and its accuracy. In recent years, to enhance the global optimization searching ability, the genetic algorithm (GA) [5] has been applied to the biotechnical field [2].

Paper Submitted 05/07/04, Accepted 09/23/04. Author for Correspondence: Hsin-Chuan.Kuo.E-mail: khc@ntou.edu.tw.

*Associate Professor, Institute of Systems Engineering and Naval Architecture, National Taiwan Ocean University, 2. Pei-Ning Road, Keelung, Taiwan 202, R.O.C.

**Professor, Institute of Systems Engineering and Naval Architecture, National, Taiwan Ocean University, 2. Pei-Ning Road, Keelung, Taiwan 202, R.O.C.

***Doctorate Candidate, Institute of Systems Engineering and Naval Architecture, National Taiwan Ocean University, 2. Pei-Ning Road, Keelung, Taiwan 202, R.O.C.
While the simplex method [10] and GA are both categorized into the primitive stage, that is, both of them are a direct search method without gradient information. Thus, it has a fast searching ability and has been widely applied to improve conditions for chemical reactions [9]. In 1962, Spendley et al. [13] proposed the basic simplex method and then, Nelder and Mead [10] further presented the modified simplex method (MSM), in which the simplex can adjust its size and shape to the response surface and this leads to the basis for further applications. It means the MSM can have the directional fast searching ability to the local optimization without the need of gradient information; however, its global optimization searching ability depends on whether the initial point is located on the nearby of the point or not. Thus, the success rate of searching minimization is not satisfied.

To improve the search capability to achieve global optimization for the simplex method, Huang et al. [6] proposed the multi-start downhill simplex method, in which the multi-start search technique was adopted to enhance the global optimization ability. In 1989, Torczon [14] further presented the multi-directional search technique to fix this problem. In this method, the simplex is generated with the multi-directional aspects, and the search technique is starting from the initial point as the center and then to expand outward for the optimal solution. Thereafter, in 2003, Xiong and Jutan [15] proposed a dynamic simplex method, which makes the size of the simplex changeable during the search process and it leads to the increasing global optimization ability of the simplex method. However, the simplex method itself has the inherent directional search and naturally results in the better solution for the local optimization during the iteration process. Thus it is inevitably trapped into the local optimal solution when the global optimization search is triggered. To own both the merits of the better local searching ability from the simplex method and the global searching ability from the evolutionary algorithm, a combined use of the two algorithms, such as Chelouah's continuous hybrid 
algorithm (CHA) [4], was developed. In this hybrid method, the GA in conjunction with the simplex method are combined together to have the better local and global optimization searching abilities simultaneously. More specifically, this hybrid method makes use of the GA to localize a "promising area" containing a global minimum and then, aimed at the area, adopts the MSM to forward the local search. The basic ideal of this hybrid method combining the GA and MSM retains the algorithms' framework but allows some parameters be determined for the GA and MSM, respectively. Therefore, the key point for resolving this issue is to find a set of optimal parameters with respect to different requirements of the problems and this needs efforts for adjusting the parameters.

Based on currently reviewed papers as above and several studies by the first author [7, 8], a hybrid of GA and MSM can be used to improve the drawbacks of the simplex method for the global optimization problems. Although the CHA is a good candidate to be adopted; however, many setting parameters are needed. Thus, we proposed a hybrid of simplex and evolution algorithm (SEA) to revisit this problem. In the SEA, only two setting parameters, population size and population renew number, are necessary and this merit is also validated through several testing examples. Basically, the SEA adopts the population evolution mechanism of the $\mathrm{GA}$, and the new individuals in the new generation are generated by the search mechanism of the MSM in place of the crossover and mutation operations of the GA. This paper, apart from the Introduction section, is organized as follows. Section 2 describes the algorithm of the MSM with several testing examples of function minimum optimization. The framework and flowchart of the SEA are included in Section 3. Comparisons of the SEA and other algorithms with respect to searching efficiency are presented in Section 4. Several concrete conclusions are made in Section 5 by recasting the reported results.

\section{THE SEARCHING CHARACTERISTICS OF THE MSM}

The search algorithm of the MSM can be described as follows. In an $n$ dimensional space, a simplex is composed of $n+1$ linearly independent vertexes, for instance, two different points can form a line in onedimensional space; three different points not at the same line can form a triangle in two-dimensional space; any four points in a different plane can form a tetrahedron. Therefore, according to the dimension of the problem like $n$ dimension, a simplex is a geometrical figure consisting, in $n$ dimensions, of $(n+1)$ points and then, the objective function of the $n+1$ points can be obtained and compared. The search direction of the objective function can be determined by using (1) reflection, $X_{r}$; (2) expansion, $X_{e}$; (3) contraction, $X_{s}$. By using this search mechanism, the search direction can be determined and the new better point can also be found for replacing the worst point among the $(n+1)$ points to form a new simplex. Owing to this search algorithm, a new simplex will contract toward the optimal point. When the contraction approaches to the convergence criterion, this replacing process stops, that is, the iteration stops. The flowchart of this algorithm for the MSM can be described as follows.

Step 1: Initial process: For an $n$-dimensional space, assuming $\varepsilon$ is the divergence criterion, $\alpha$ the reflection coefficient, $\gamma$ the expansion coefficient and $\beta$ the contraction coefficient. For a given initial point $X_{o}$, the other $n$ vertexes can be found by starting from $X_{o}$ along the axis direction $e_{i}(i=1,2, \ldots, n)$ with the side length of $h$ : $X_{j}(j=2,3, \ldots \ldots, n+1)$.

$X_{j}=X_{o}+h^{*} e_{i}(j=2,3, \ldots, n+1)$,

in which $e_{i}$ represents the unit vector of the $i$ - th axis and $h$ represents the side length.

Step 2: Evaluating process: To evaluate the priority of the $(n+1)$ vertexes in orders, for instance, to search for the minimum optimization, the smaller the value of the vertex is the better and the larger is the worse.

Step 3: New point generation process: By using the reflection, expansion and contraction mechanisms, the new point can be generated as shown in Fig. 1. In this figure, $X_{l}$ is the best choice, then $X_{g}$ is the second and the $X_{h}$ is the worst. Besides, $X_{c}$ represents the centroid of the simplex excluding of the point, $X_{h}$. Thus, we can have

$X_{c}=\frac{1}{n} \sum_{i=1, i \neq h}^{n+1} X_{i}$

and

(1) Reflection: $X_{r}=X_{c}+\alpha\left(X_{c}-X_{h}\right)$, as shown in Fig. 1(a);

(2) Expansion: $X_{e}=X_{c}+\gamma\left(X_{r}-X_{c}\right)$, as shown in Fig. 1(b);

(3) Contraction: Condition (a): $X_{s}=X_{c}+\beta\left(X_{h}-\right.$ $X_{c}$ ), as shown in Fig. 1(c); Condition (b): Contract to the point $X_{l}$ as shown in Fig. 1(d).

Step 4: If the average mean square distance of all points to the centroid of the simplex approaches to the convergence threshold, $\varepsilon$, then stop searching. Thus, one can choose the best point as the 
optimal solution; otherwise, one has to skip to step 2 for iteration and the convergence criterion can be expressed as

$$
\left\{\frac{1}{n+1} \sum_{j=1}^{n+1}\left[f\left(X_{j}\right)-f\left(X_{c}\right)\right]^{2}\right\}^{\frac{1}{2}} \leq \varepsilon .
$$

To verify searching characteristics of the MSM, a two-dimensional single-minimum function $f_{1}$, the Rosenbrock function, and the two-dimensional multiminimum function $f_{2}$ (all these testing functions are listed in the appendix section for quick reference) are chosen as the testing examples, respectively; the former $f_{1}$ is used to evaluate the local searching ability of the MSM, while the latter $f_{2}$ is for global searching ability of the MSM. Here, the parameters of the MSM are given as: the side length, $\mathrm{ST}$, is equal to 1 ; the reflection coefficient $\alpha=2$; the expansion coefficient $\gamma=2$; contraction coefficient $\beta=0.5$; the convergence criterion $\varepsilon$ is set to be $1 \times 10^{-4}$. Besides, five different sets of initial points are given for these testing examples.

All the results of the testing examples are listed in Table 1 and Table 2. In this table, NOFE represents the

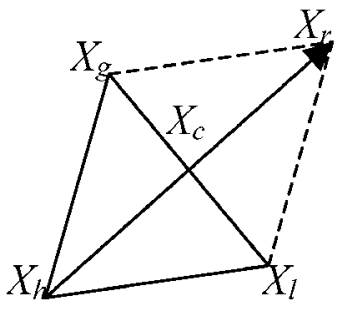

(a)

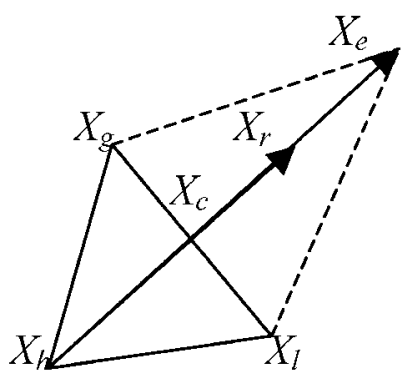

(b)

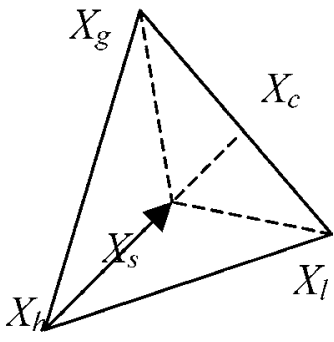

(c)

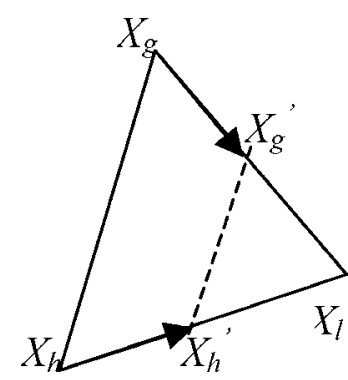

(d)
Fig. 1. Moving schemes in the MSM: (a) reflection, (b) expansion, (c) contraction, and (d) multi- contraction. total numbers of the objective function evaluations being called when searching. It is found that when the MSM is applied to the $f_{1}$, the minimum solutions can be reached for various initial points and the average error to the analytical solution is $3.8122 \times 10^{-7}$ only. Basically, for this single-minimum function $f_{1}$, the global minimum is located in the middle of the bananashape long and narrow valley [4] and our search process also shows the similar condition with the final result at an accepted convergence. For the $f_{2}$ testing condition, five cases are setting with five initial points given at the nearby of the five local minima, respectively. Testing results show that the global minimum can be yielded only available for the third case and for the other cases, the local minimum solutions at the nearby of the initial points can be reached. It means that generally the MSM can be used to search for the local minimum optimization for the multi-minimum function for different initial points.

As results shown above, the global minimum optimization searching ability of the MSM is not good enough such that a trial of enlarging the initial side length is conducted for testing whether a larger simplex can help the MSM skip out the local optimal solution as shown in Fig. 2. Again taking $f_{2}$ as testing example, the calculation results are listed in Table 3. It is shown that when the side length has been adjusted to be 5 and 10 , respectively, cases 2 and 4 can yield the local optimal solution nearby the initial points; while cases 1,3 , and 5 can still reach other local optimal solution. This

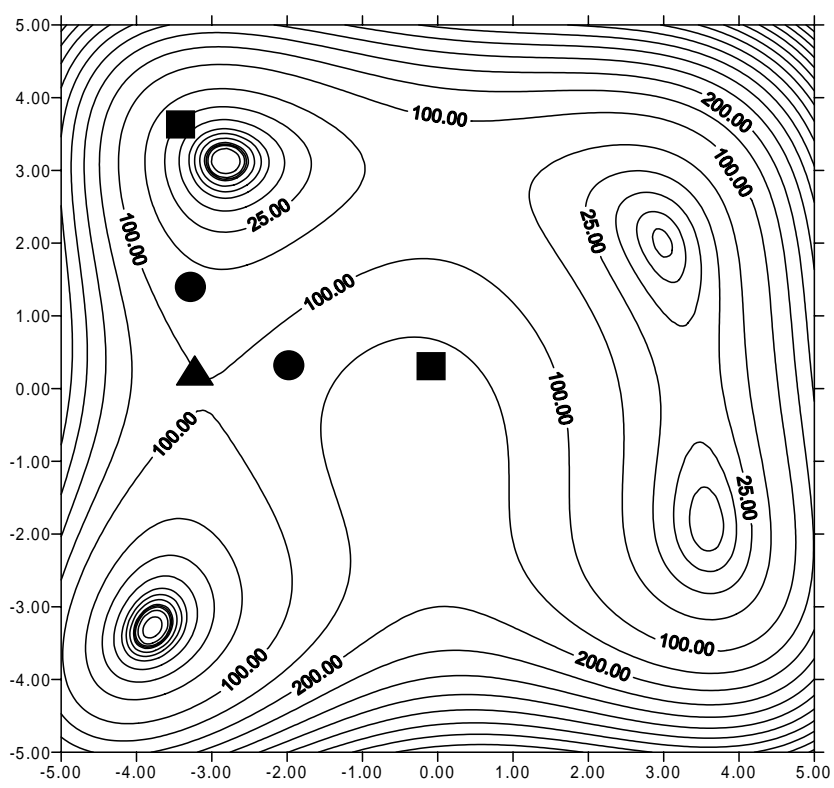

Fig. 2. The initial different simplexes in $\mathbf{2}$ dimensions: $\boldsymbol{\Delta}$ : is the initial point; $\bigcirc$ : other vertices produced by using a smaller side length; $\square$ : other vertices produced by using a larger side length. 
Table 1. Optimization results of the MSM with different initial points for $f_{l}(x)$

\begin{tabular}{cccccc}
\hline$f_{1}(x) /$ Case & 1 & 2 & 3 & 4 & 5 \\
\hline Initial point & $(-3,3)$ & $(3,3)$ & $(-3,-3)$ & $(3,-3)$ & $(1,-1)$ \\
Solution and function & $(1,1)$ & $(1,1)$ & $(1,1)$ & $(1,1)$ & $(1,1)$ \\
value of local minimum* & 0 & 0 & 0 & 0 & 0 \\
NOFE** & 203 & 193 & 185 & 163 & 236 \\
The final solution & $(0.999,0.999)$ & $(0.999,0.999)$ & $(0.999,0.998)$ & $(1.000,1.001)$ & $(1.000,1.000)$ \\
The final function value & $2.84 \mathrm{E}-07$ & $3.753 \mathrm{E}-07$ & $4.332 \mathrm{E}-07$ & $7.95 \mathrm{E}-07$ & $4.39 \mathrm{E}-07$ \\
\hline
\end{tabular}

* Local minimum: the initial points of the five cases are located in the neighborhoods of the local minimum.

**NOFE: the total number of the objective function evaluations being called when searching.

Table 2. Optimization results of the MSM with different initial points for $f_{2}(x)$

\begin{tabular}{cccccc}
\hline$f_{2}(x) /$ Case & 1 & 2 & 3 & 4 & 5 \\
\hline Initial point & $(-1,4)$ & $(2,2)$ & $(-3,-2)$ & $(3,-2)$ & $(0,-1)$ \\
Solution and function & $(-2.820,3.131)$ & $(2.983,2.009)$ & $(-3.788,-3.246)$ & $(3.574,-1.845)$ & \\
value of local minimum* & -2.813 & 2.990 & -3.780 & 3.579 & 85 \\
NOFE** & 88 & 77 & 82 & 72 & $(2.984,2.011)$ \\
The final solution & $(-2.822,3.132)$ & $(2.985,2.007)$ & $(-3.789,-3.287)$ & $(3.579,-1.847)$ & 2.9919 \\
The final function value & -2.812 & 2.9920 & -3.7838 & 3.5797 & 2.78 \\
\hline
\end{tabular}

* Local minimum: the initial points of the five cases are located in the neighborhoods of the local minima.

**NOFE: the total number of the objective function evaluations being called when searching.

Table 3. Optimization results of the MSM with different side length, ST, and initial point for $f_{2}(x)$

\begin{tabular}{|c|c|c|c|c|c|}
\hline$f_{2}(x)$ /initial point & $(-1,4)$ & $(2,2)$ & $(-3,-2)$ & $(3,-2)$ & $(0,-1)$ \\
\hline Solution and function & $(-2.820,3.131)$ & $(2.983,2.009)$ & $(-3.788,-3.246)$ & $(3.574,-1.845)$ & $(2.983,2.009)$ \\
\hline value of local minimum & -2.813 & 2.990 & -3.780 & 3.579 & 2.990 \\
\hline $\begin{array}{l}\text { The final function } \\
\text { value, } \mathrm{ST}=1\end{array}$ & -2.812 & 2.992 & -3.783 & 3.579 & 2.991 \\
\hline $\begin{array}{l}\text { The final function } \\
\text { value, } \mathrm{ST}=5\end{array}$ & 2.992 & 2.992 & -2.812 & 3.579 & 3.579 \\
\hline $\begin{array}{c}\text { The final function } \\
\text { value, } \mathrm{ST}=10\end{array}$ & 3.579 & 2.991 & 2.992 & 3.579 & -2.812 \\
\hline
\end{tabular}

phenomenon shows that the MSM can have the ability to skip out the local optimal solution. Finally, initial points of generating randomly are also conducted to find the global minimum for calculating the success rate of this algorithm. The success rate is defined as the number of reaching the global minimum per 100 times of random search. The results are shown in Table 4. In this table, the best success rate appears in the case of ST $=1$ and approaches to $17 \%$ with an average $N O F E$ value of 93; while for the other cases, the success rates is $10 \%$ approximately. It is shown that the randomly generating initial points in conjunction with a simplex of polyhedron with different side lengths can possibly search for the global minimum optimization. In addition, with more given initial points the success rate of yielding the optimal solution can increase.

\section{THE HYBRID ALGORITHM OF EVOLUTION AND SIMPLEX METHODS}

The proposed hybrid algorithm of evolution and simplex methods is a simplex-based evolutionary algorithm (SEA). The SEA adopts the population evolution process of the GA and introduces the searching mechanism of the MSM for generating the new generation. With the Darwin's theory of evolution, "Survival at the fittest or fail at the non-fittest", the proposed SEA combines the evolution method and the MSM with 
Table 4. Optimization results of the MSM with some different side length, ST, for $f_{2}(x)$

\begin{tabular}{cccccc}
\hline The side length of simplex & $\mathrm{ST}=1$ & $\mathrm{ST}=3$ & $\mathrm{ST}=5$ & $\mathrm{ST}=7$ & $\mathrm{ST}=10$ \\
\hline Success rate $(\%)$ & 17 & 14 & 8 & 10 & 7 \\
NOFE $E_{a v}$ & 93 & 107 & 100 & 124 & 129 \\
The final function value & -3.783 & -3.783 & -3.783 & -3.783 & -3.783 \\
Global minimum of $f_{2}(x)$ & -3.783 & -3.783 & -3.783 & -3.783 & -3.783 \\
\hline
\end{tabular}

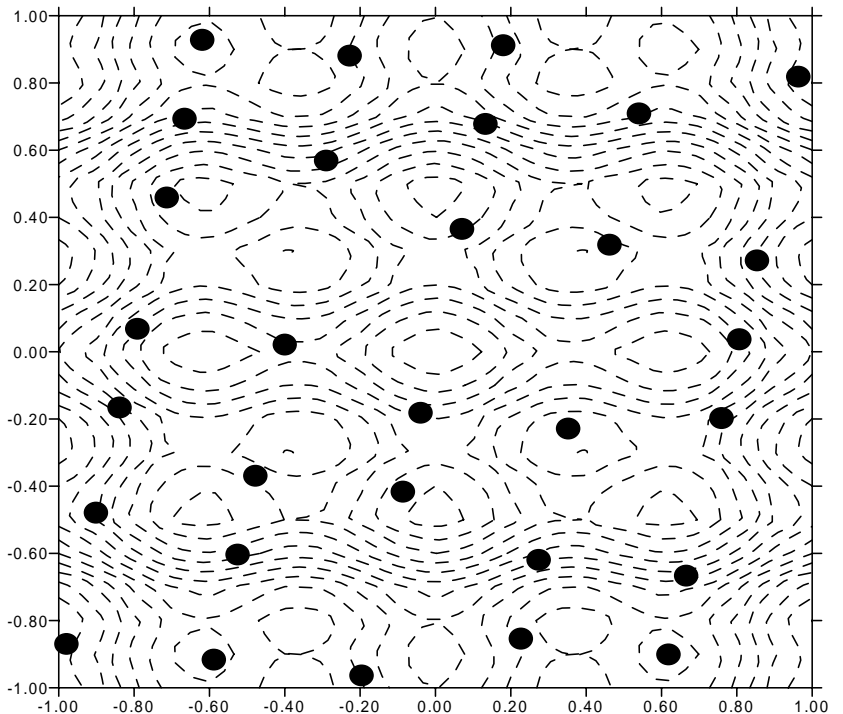

Fig. 3. The uniform distribution of the initial population ( 30 individuals) $f_{6}(x)$ for in the searching space.

respect to their own merits to deal with the global minimum optimization.

\section{Algorithm of the SEA}

Basically, two parameters need to be setup for the SEA in advance: 1 . the population size: $(n+1)$ of the individuals is chosen for the initial generation with a uniform format; 2 . the renew number: how many number of the new individuals is needed being called the renew number, for instance, if there need five renew individuals for a new generation, then we call the renew number as 5. As for the generation scheme of the initial population, the neighborhood concept is adopted, that is, we take the individual $X$ as the center and the radius $r$ to form a circle, and then, the generated individual $X^{\prime}$ should satisfy the condition of $\left\|X^{\prime}-X\right\| \geq r$ such that the initial population can be uniformly distributed over the searching space, as shown in Fig. 3. The generation schemes of the new population for the SEA can be described as follows.

(1) Choosing an individual of the population randomly as the worse point $X_{h}$, (may not the worst of the whole population) and calculating the centroid, $X_{c e n}$, of all individuals which are better then $X_{h}$. By using the search direction that the $X_{h}$ faces toward the $X_{c e n}$, the new individual $X_{n}$ can be obtained. That is,

$$
\begin{aligned}
& \text { Search direction: } S_{1}=X_{c e n}-X_{h} \text {, } \\
& \text { New individual: } X_{n}=X_{c e n}+\alpha \cdot S_{1} \text {. }
\end{aligned}
$$

(2) Choosing two individuals of the population randomly and evaluating which one is better. The new individual $X_{n}$ can be yielded by using the search direction that the worse $X_{h}$ faces toward the better $X_{g}$. That is

$$
\begin{aligned}
& \text { Search direction: } S_{2}=X_{g}-X_{h}, \\
& \text { New individual: } X_{n}=X_{g}+\alpha \cdot S_{2} \text {, }
\end{aligned}
$$

in which $\alpha$ is taken 0.618 . The two search directions are shown in Fig. 4.

In summary, the algorithm for the SEA can be expressed as:

Step 1: Initial process: To determine the population size, the renew number and the searching iteration number and then, to generate the initial population with a uniform format.

Step 2: Evaluating process: To evaluate the fitness of the individuals of the population and reordering according to their fitness.

Step 3: New generation process: In the evaluating process, those individuals of higher fittest survive and knock the lowers out.

Step 4: Determining the search direction: By using the two generation schemes as mentioned to determine the search direction $S_{1}$ and $S_{2}$ and then, to produce new individuals in place of the worse individuals.

Step 5: To judge whether the error between the analytical minimum of the function and the numerical solution approaches to the converging criterion; if it reaches, then stop searching. Thus, one can choose the best individual as the optimal solution. Otherwise, to judge whether the num- 


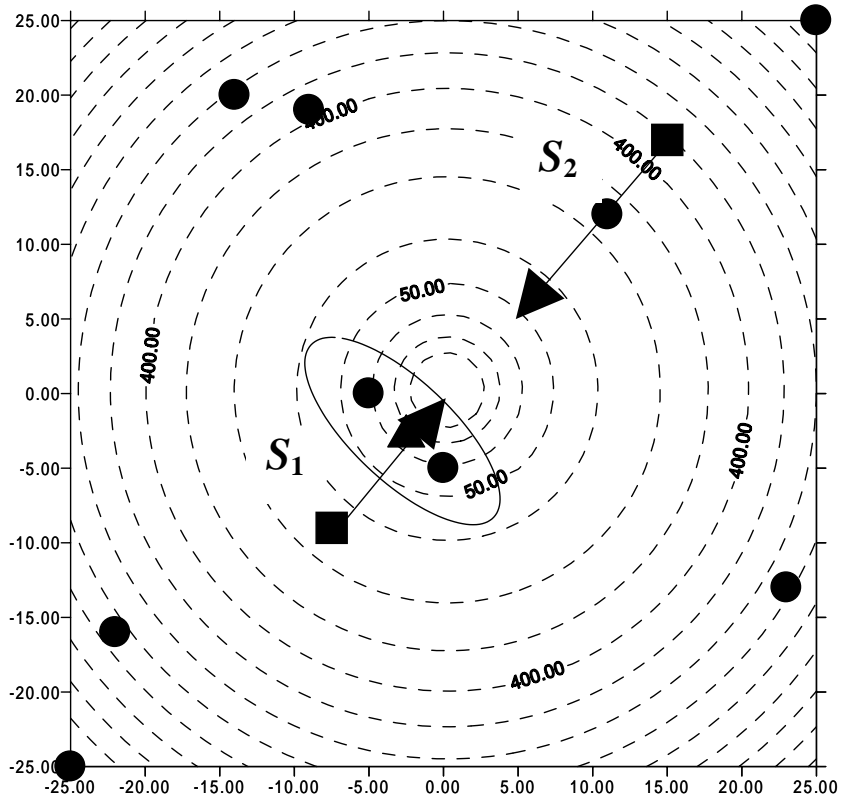

Fig. 4. The search direction $S_{i}, i=1,2$ : (1) $\Delta$ is the centroid of the individuals in the circle, $S_{1}$ is from the $\mathbf{\square}$ faces toward the $\mathbf{\Delta}$; (2) $S_{2}$ is from the $\square$ faces toward the $\bullet$.

ber of new individuals is equal to the renew number, if it does, then skip to Step 2, otherwise, skip to Step 3.

\section{Searching track of the SEA}

As previous section shown, if the side length of a simplex enlarge for the MSM, then a larger simplex can help the MSM skip out the local optimal solution. To validate such characteristics of the SEA, again the multi-minimum function of $f_{2}$ is used to test the global minimum optimization searching ability of the SEA and an observation of the searching track, which can show the searching characteristic and the process of population revolution, is also conducted. Every ten generations is adopted for recording the population position to observe how the population move and yield the final minimum. In this case, the population size is 30 and the renew number is 21 , which is $70 \%$ of the population size. Thus, one can find how the SEA makes the population with individuals jump out the local minimum searching and then to yield the global optimal solution.

Fig. 5 shows the results of several generations of populations in the case of $f_{2}$ function for the SEA. In this figure, when the population evolves in the stage of initial generation, the population individuals appear at the nearby of the four minimum values of the contours. When it further evolves into the $20^{\text {th }}$ generation, some individuals of higher values gradually move (so-called the renew process) to up-right area of local minimum in the contours; however, several individuals still exist in the lower-left area due to their fitness. Since those individuals in the up-right area are more than those in the lower-left area such that the opportunity of generating new individuals in that area will increase. Even so, the individuals of the whole population are still sparse and it means these individuals can move to other search space for yielding the global optimization. Besides, during the evolution from the $30^{\text {th }}$ to $110^{\text {th }}$ generation, the individuals start to move to the lower-left area of global optimization from the up-right area of local minimum gradually over a contour peak area of the middle part of the searching space. More specifically, at the $30^{\text {th }}$ generation, those individuals located at the nearby valley of the up-right area gradually expand, move and finally contract at the nearby valley of the lower-left area at the $110^{\text {th }}$ generation. Thereafter, at the $120^{\text {th }}$ generation, most of the individual center to the valley of global minimum of the lower-left area, but still few individuals appear elsewhere. Anyway, the population finally yields the global optimization and the search can stop here.

As one can find the population moving track as shown in Fig. 5, individuals of the worse fitness will be filtered out at each generation revolution and are replaced by the better individuals. While individuals with the better fitness will survive and thus, it does not makes more and more individuals contract to the local minimum until another individuals of the population can find the other better local minimum. Therefore, the SEA can have the expansion and contraction operations to search for the global minimum optimization repeatedly. Besides, during the search process of the SEA where happens the contraction phenomenon represents the existence of the local minimum. Moreover, the SEA can have the ability to jump out the local minimum and not continue to expand for searching until the population contracts at the highest fitness of the global minimum.

\section{TESTING AND DISCUSSION ON THE SEARCHING EFFICIENCY OF THE SEA}

\section{Testing function examples of the SEA}

Eight different kinds of functions listed in the appendix are adopted for testing the success rate of searching for the global optimization of the SEA. The convergence criterion is defined as [1]

$$
\left|O B J_{S E A}-O B J_{A N A L}\right| \leq 10^{-4},
$$




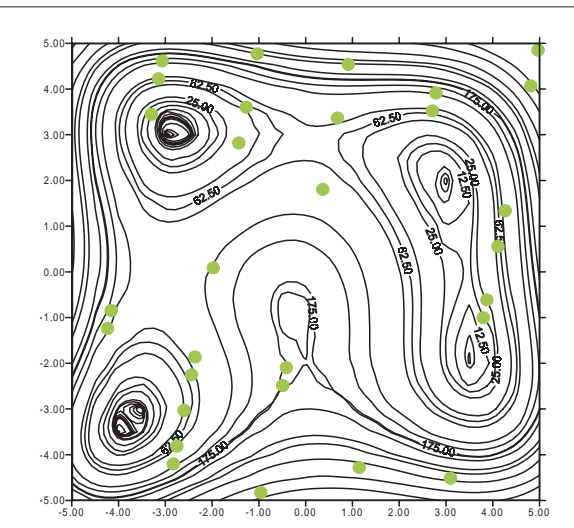

(a) The initial generation

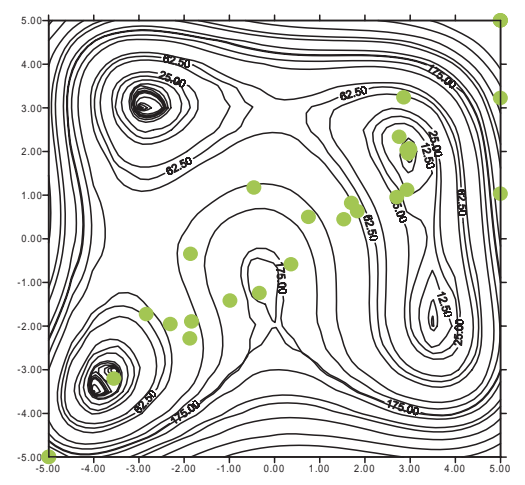

(d) The 40th generation

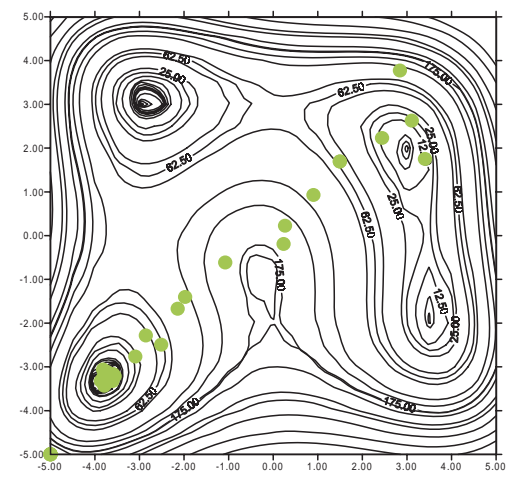

(g) The 110th generation

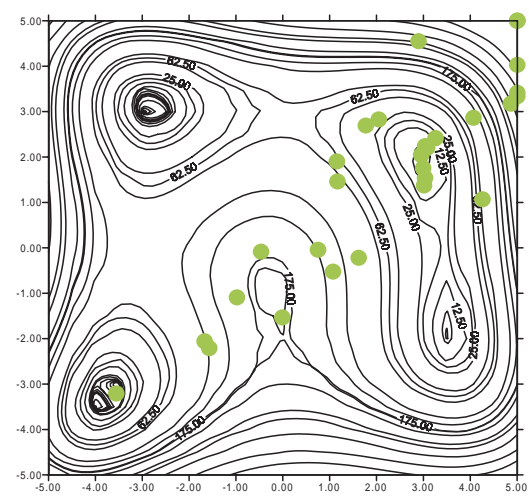

(b) The 20th generation

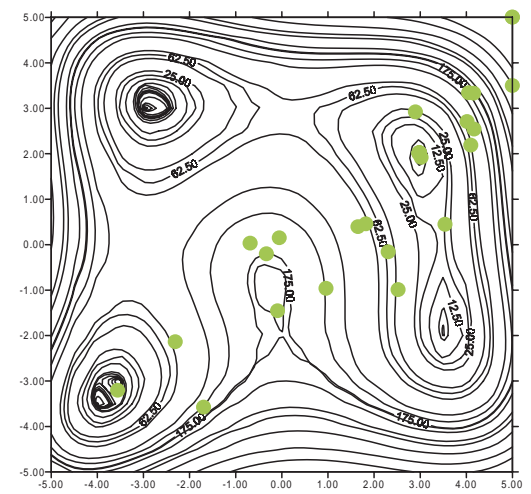

(e) The 80th generation

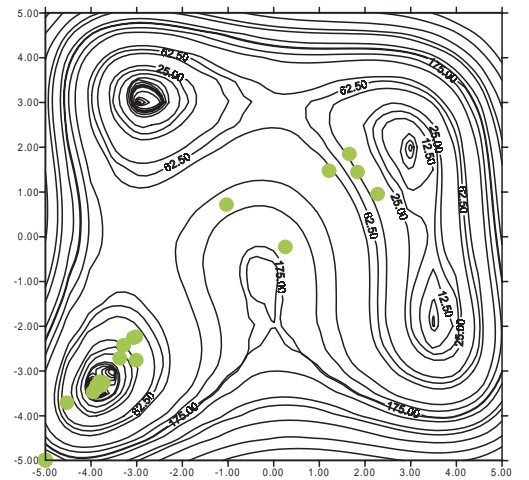

(h) The 120th generation

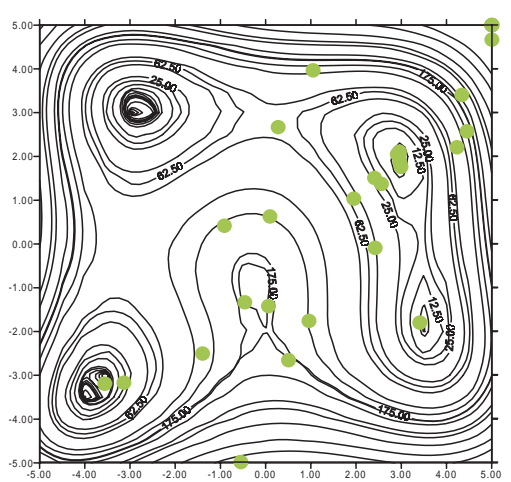

(c) The 30th generation

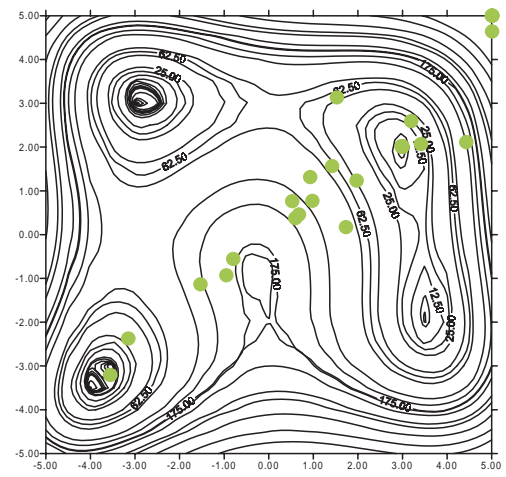

(f) The 90th generation

Fig. 5. The moving trajectory of the population during generation, the population size is 30 . in which $O B J_{S E A}$ represents the searched global optimum by the SEA and $O B J_{A N A L}$ represents the analytical solution of the optimization problem. All the testing results are listed in Table 5 and the success rate is defined as how many cases of searching results satisfying the convergence criterion in 100 searching cases. In Table 5, the symbol NOFE $E_{a v}$ denotes the average number of objective function evaluations for the success searching cases, and the average error $\varepsilon_{a v}$ is defined as the averaging value of the difference between the searched optimal solution and analytical solution for the success searching cases. In addition, the testing functions including two single- minimum functions and six multi-minimum functions are used and as shown in this table, the searching success rates of the eight testing functions are all above $93 \%$ and the average success rate of all testing function is $98 \%$. Therefore, no matter for the single- or multi- minimum functions, the SEA shows its high searching success rate for improving the global searching ability of the MSM. 


\section{Comparison of the SEA and other evolution algorithms}

For further validating the proposed SEA, five different evolution algorithms for the global minimum search [1, 3-4, 7-8] are adopted to search for the NOFE for the efficiency comparison with the SEA. Here, six different kinds of testing functions are chosen and most of them are two-dimensional multi-minimum functions as shown in the appendix. The same convergence criterion, as shown above, is adopted again for testing [1] and all testing results are shown in Table 6. Among all used algorithms, those related with the GA are SEA, the CHA, the continuous genetic algorithm (CGA) and the real-value coding genetic algorithm (RCGA). For the CHA [4], the GA is adopted for possible global optimum position and the MSM is introduced for the local search in the searched global optimum positions. As for the CGA [3], the discrete variables in the binary code are replaced by the continuous variables in the real code for the GA. For the RCGA, the initial population is generated by using the entropy theory and a search technique for finding the new smaller search space in the neighborhood of the global optimum is adopted. As for the other two algorithms, the basic idea of the continuous Tabu search (CTS) [11] is to put the searched local minimum solutions into the Tabu list such that it

Table 5. Optimization results of the SEA for eight testing functions

\begin{tabular}{ccrc}
\hline Function & $\begin{array}{c}\text { Success } \\
\text { rate }(\%)\end{array}$ & NOFE & $\varepsilon_{a v}$ \\
\hline$f_{1}(x), \mathrm{R}_{2}$ & 97 & 266 & $7.11 \mathrm{E}-06$ \\
$f_{2}(x), \mathrm{MH}$ & 93 & 1136 & $5.22 \mathrm{E}-06$ \\
$f_{3}(x), \mathrm{ES}$ & 97 & 197 & $4.42 \mathrm{E}-05$ \\
$f_{4}(x), \mathrm{RC}$ & 100 & 272 & $6.40 \mathrm{E}-05$ \\
$f_{5}(x), \mathrm{Z}_{2}$ & 97 & 90 & $5.22 \mathrm{E}-05$ \\
$f_{6}(x), \mathrm{B}_{2}$ & 100 & 258 & $2.82 \mathrm{E}-05$ \\
$f_{7}(x), \mathrm{SH}$ & 100 & 420 & $1.97 \mathrm{E}-05$ \\
$f_{8}(x), \mathrm{DJ}$ & 100 & 136 & $4.21 \mathrm{E}-05$ \\
\hline
\end{tabular}

avoids the next iteration approaching to these local minimum solutions. As for the enhanced simulated annealing (ESA) [12], its search technique is easy to implement, requires little expert knowledge and is not memory intensive.

In Table 6, a comparison for the searching efficiency of the SEA with those quite similar search algorithms, such as the SEA, CHA, RCGA and CGA, is conduced and the results show that for the single-minimum function $f_{1}(x), R_{2}$, the searching efficiency of $N O F E=266$ for the SEA is the best and the second is $\mathrm{CHA}$ with $N O F E=459$. For the multi-minimum functions results show that the SEA, in general, has the dominant searching efficiency as compared with other search algorithms and the second is the CHA. Basically, these two algorithms both adopt the global search ability of the GA in conjunction with the local search ability of the MSM; however, what differences for these two algorithms are: the CHA first conducts the crossover and mutation mechanisms of the GA to generate the new individuals and to explore the possible positions of the global optimal solutions and then, these possible positions are taken as the initial points for the MSM to search for the local optimal solution; while the SEA adopts the direction-based search mechanism of the MSM to generate the new individuals. In addition, the necessary setting parameters of search algorithms are also different, that is, the CHA needs eight parameters while the SEA only need two such that it makes the SEA more convenient to implement for the optimal search. Finally, a comparison of the SEA with the CTS and the ESA also shows that the SEA is superior to the other two algorithms no matter for the single- or multi-minimum functions.

\section{CONCLUSIONS}

Several concrete conclusions can be drawn as follows:

1. The proposed hybrid method, SEA, adopts the search technique of the simplex method and introduces the

Table 6. A comparison of the searching efficiency of the NOFE for the SEA with other algorithms

\begin{tabular}{ccccccc}
\hline $\begin{array}{l}\text { Method/ } \\
\text { Function }\end{array}$ & $\begin{array}{c}\text { SEA } \\
\text { [present] }\end{array}$ & $\begin{array}{c}\text { CHA } \\
{[4]}\end{array}$ & $\begin{array}{c}\text { CGA } \\
{[3]}\end{array}$ & $\begin{array}{c}\text { RCGA } \\
{[1]}\end{array}$ & $\begin{array}{c}\text { CTS } \\
{[11]}\end{array}$ & $\begin{array}{c}\text { ESA } \\
{[12]}\end{array}$ \\
\hline$f_{1}(x), \mathrm{R}_{2}$ & 266 & 459 & 960 & 596 & 1616 & 796 \\
$f_{2}(x), \mathrm{ES}$ & 197 & 952 & 1504 & 642 & - & - \\
$f_{3}(x), \mathrm{RC}$ & 272 & 295 & 620 & 490 & 668 & \\
$f_{4}(x), \mathrm{Z}_{2}$ & 90 & 215 & 620 & 437 & 689 & -15820 \\
$f_{5}(x), \mathrm{B}_{2}$ & 258 & 132 & - & - & - & - \\
$f_{6}(x), \mathrm{SH}$ & 420 & 345 & 575 & - & - & - \\
\hline
\end{tabular}


concept of all population information and thus, one can have the greater populations for searching. Besides, the SEA offers two strategies of search directions, $S_{1}$ and $S_{2}$, for generating new individual to find the global minimum solution with respective generation.

2. From the observation of the population moving, the SEA has the expansion and contraction operations to search for the global minimum optimization. Further more, the SEA can search other local minimum solutions during the search process so that they can provide designer with more information to make policy beneficially.

3. The average success rate of the SEA in eight testing cases for searching global minimum approaches to 98\% and thus, it proves that the SEA have an excellent searching ability for the global optimal solution.

4. For the calculating efficiency of the NOEE index, a comparison of the SEA with those similar search algorithms of GA, such as the SEA, CHA, RCGA and CGA, show that the proposed SEA is the best and the second is CHA. A comparison of the SEA with the CTS and the ESA also shows that the SEA is superior to the other two algorithms.

5. Finally, an evaluating mechanism of population moving stability, which can be used to evaluate parameters of SEA, such as the population size and the renew number, and deal with the dynamics information of individual moving more effectively, is suggested for future study of the SEA.

\section{REFERENCES}

1. Bessaou, M. and Siarry, P., "A Genetic Algorithm with Real-Value Coding to Optimize Multimodal Continuous Functions," Struct. Multidisc. Optim., Vol. 23, pp. 63-74 (2001).

2. Braaten, O., Rodningen, O.K., Nordal, I., and Leren, P. T., "The Genetic Algorithm Applied to Haplotype Data at the LDL Receptor Locus," Comput. Methods Prog. Biomed., Vol. 61, No. 1, pp. 1-9 (2000).

3. Chelouah, R. and Siarry, P., "A Continuous Genetic Algorithm Designed for the Global Optimization of Multimodal Functions," J. Heuristics, Vol. 6, pp. 191213 (2000).

4. Chelouah, R. and Siarry, P., "Genetic and Nelder-Mead Algorithms Hybridized for a More Accurate Global Optimization of Continuous Multiminima Functions," Eur. J. Oper. Res., Vol. 148, pp. 335-348 (2003).

5. Holland, J.H., Adaption in Natural and Artificial Systems, The University of Michigan Press, Ann Arbor (1975).

6. Huang, M., Aine, C.J., Supek, S., Best, E., Ranken, D., and Flynn, E.R., "Multi-Start Downhill Simplex Method for Spatial-temporal Source Localization in Magneto
Encephalography," Electroencephalogr. Clinical Neurophysiol. Evoked Potent. Sect., Vol. 108, No. 1, pp. 32-44 (1998).

7. Kuo, H.C. and Kuan, C.C., "A Modified Simplex Search Method for Structural Optimizations," J. Marit. Res., Vol. 8, pp. 1-18 (1999).

8. Kuo, H.C. and Kuan, C.C., "A Random Search Algorithm for Multi-Modal Problems," J. Soc. Naval Archit. Marine Eng., Vol. 19, No. 4, pp. 33-40 (2000).

9. Matsumoto, T., Du, H., and Lindsey, S.J., “A Parallel Simplex Search Method for Use with an Automated Chemistry Workstation," Chemometrics Intel. Lab. Syst., Vol. 62, No. 2, pp. 129-147 (2002).

10. Nelder, J.A. and Mead, R., "A Simplex Method for Function Minimization," Comput. J., Vol. 7, pp. 308313 (1965).

11. Siarry, P. and Berthiau, G., "Fitting of Tabu Search to Optimize Functions of Continuous Variables," Int. J. Numer. Methods Eng., Vol. 40, pp. $2449-2457$ (1997).

12. Siarry, P., Berthiau, G., Durbin, F., and Haussy, J., "Enhanced Simulated Annealing for Globally Minimizing Functions of Many Continuous Variables," ACM Trans. Math. Softw., Vol. 23, No. 2, pp. 209-228 (1997).

13. Spendley, W., Hext, G.R., and Hims-Worth, F.R., "Sequential Application of Simplex Designs in Optimization and Evolutionary Operation," Technometrics, Vol. 4, pp. 441-461 (1962).

14. Torczon, V.J., "Multi-Directional Search: A Direct Search Algorithm for Parallel Machines," Ph.D. Dissertation, Department of Mathematical Sciences, Rice University, Houston (1989).

15. Xiong, Q. and Jutan, A., "Continuous Optimization Using a Dynamic Simplex Method," Chem. Eng. Sci., Vol. 58, pp. 3817-3828 (2003).

\section{APPENDIX: LIST OF TEST FUNCTIONS}

$f_{1}:$ Rosenbrock $\left(R_{2}\right)$ (2 variables)

$f_{1}(x)=100\left(x_{1}^{2}-x_{2}\right)^{2}+\left(x_{1}-1\right)^{2}$

Search domain: $-5, x_{i}<5, i=1,2,3, \ldots, N$;

The global minimum: $x^{*}=(1, \ldots, 1), f_{l}\left(x^{*}\right)=0$.

$f_{2}$ : Modified Himmelblau Function (MH) (2 variables)

$f_{2}(x)=\left(x_{2}+x_{1}^{2}-11\right)^{2}+\left(x_{1}+x_{2}^{2}-7\right)^{2}+x_{1}$

Search domain: $-5<x_{i}<5, i=1,2$;

Number of local minima $=3$;

The global minimum: $x^{*}=(-3.788,-3.246)$, 
$f_{2}\left(x^{*}\right)=-3.78$.

$f_{3}: \operatorname{Easom}(E S)$ (2 variables)

$f_{3}(x)=-\cos \left(x_{1}\right) \cdot \cos \left(x_{2}\right) \cdot \exp \left(-\left(\left(x_{1}-\pi\right)^{2}+\left(x_{2}-\pi\right)^{2}\right)\right)$

Search domain: $-100<x_{i}<100, i=1,2$;

Multiple local minima (exact number unspecified in the literatures);

The global minimum: $x^{*}=(-3.788,-3.246)$, $f_{3}\left(x^{*}\right)=-1$.

$f_{4}:$ Branin $R \operatorname{COS}(R C)$ (2 variables)

$f_{4}(x)=\left(x_{2}-\left(5 /\left(4 \pi^{2}\right)\right) x_{1}^{2}+(5 / \pi) x_{1}-6\right)^{2}$

$+10(1-(1 / 8 \pi)) \cos \left(x_{1}\right)+10$

Search domain: $-5<x_{i}<10, i=1,2$;

Three global minimum solutions: $x^{*}=(-\pi, 12.275)$, $(\pi, 2.275),(9.42478,2.475)$,

$f_{4}\left(x^{*}\right)=0.39788$.

$f_{5}: \operatorname{Zakharov}\left(Z_{n}\right)$ (2 variables)

$f_{5}(x)=\left(\sum_{i=1}^{n} x_{i}^{2}\right)+\left(\sum_{i=1}^{n} 0.5 i \cdot x_{i}\right)^{2}+\left(\sum_{i=1}^{n} 0.5 i \cdot x_{i}\right)^{4}$

Search domain: $-5<x_{i}<10, i=1,2$;

Multiple local minima (exact number unspecified in the literatures);

The global minimum: $x^{*}=(0,0), f_{5}\left(x^{*}\right)=0$.

$f_{6}: B 2$ (2 variables)

$f_{6}(x)=x_{1}^{2}+2 x_{2}^{2}-0.3 \cos \left(3 \pi x_{1}\right)-0.4 \cos \left(4 \pi x_{2}\right)+0.7$

Search domain: $-100<x_{i}<100, i=1,2$;

Multiple local minima (exact number unspecified in the literatures);

The global minimum: $x^{*}=(0,0), f_{6}\left(x^{*}\right)=0$.

$f_{7}$ : Shubert (SH) (2 variables)

$f_{7}(x)=\left\{\sum_{j=1}^{5} j \cdot \cos \left[(j+1) x_{1}+j\right]\right\} \times\left\{\sum_{j=1}^{5} j \cdot \cos \left[(j+1) x_{2}+j\right]\right\}$

Search domain: $-10<x_{i}<10, i=1,2$;

760 local minima; 18 global optimal solutions;

The global minimum: $f_{7}\left(x^{*}\right)=-186.7309$.

$f_{8}$ : De Joung F1(DJ) (3 variables)

$f_{8}(x)=x_{1}^{2}+x_{2}^{2}+x_{3}^{2}$

Search domain: $-5.12<x_{i}<5.12, i=1,2,3$;

The global minimum: $x^{*}=(0,0,0), f_{8}\left(x^{*}\right)=0$. 